\title{
Ulnar Nerve Compression in Guyon's Canal by Ulnar Artery Cystic Adventitial Disease: A Case Report
}

\author{
João Ribeiro Afonso ${ }^{a}$ João Carvas ${ }^{b}$ Miguel Quesado ${ }^{a}$ \\ João Vasconcelos ${ }^{c}$ José Vidoedo ${ }^{c}$ Daniel Brás Lopes ${ }^{a} \quad$ Clyde Viamonte $^{a}$ \\ aDepartment of Traumatology and Orthopaedics, Centro Hospitalar Tâmega e Sousa, \\ Penafiel, Portugal; ${ }^{b}$ General Surgery Department, Unidade Local de Saúde do Nordeste \\ Transmontano, Bragança, Portugal; 'Department of Angiology and Vascular Surgery, Centro \\ Hospitalar Tâmega e Sousa, Penafiel, Portugal
}

\section{Keywords}

Adventitial cystic disease · Guyon's canal decompression · Ulnar neuropathy · Wrist

\begin{abstract}
Cystic adventitial disease is a condition where mucinous cyst(s) develop within the adventitia of blood vessels, especially arteries. The most affected vessel is the popliteal artery while the upper limb vasculature is seldom involved. To our knowledge, there are only 2 articles reporting this disease in the ulnar artery. We present a case of a 52-year-old female patient, a manual worker in a clothing factory, with a month history of increasing pain in her right wrist and gradual weakness that incapacitated her for work activities. She was finally treated surgically and an adventitial cyst of the ulnar artery compressing the ulnar nerve was diagnosed.
\end{abstract}

\footnotetext{
(C) 2021 The Author(s).

Published by S. Karger AG, Basel
}

\section{Introduction}

Cystic adventitial disease (CAD) is a rare benign vascular lesion, which predominantly affects arteries, being characterized by progressive accumulation of mucinous material in the adventitia of the vessel wall [1]. This rare entity was first described in 1947 by Atkins et al. [2] and since then multiple case reports have been published, mainly affecting arteries of the lower limb, with involvement of upper extremity vessels being extremely rare [1]. Usually the reported symptoms are related to the narrowed lumen of the vessel or by the compressive effect on the adjacent neurovascular bundle. We report a case of ulnar artery CAD at Guyon's canal that presented as ulnar tunnel syndrome.

\section{Karger ${ }^{\prime \prime}=$}


Case Reports in Orthopedic Research
Case Rep Orthop Res 2021;4:33-38

\begin{tabular}{l|l}
\hline DOI: 10.1159/000512321 & ( 2021 The Author(s). Published by S. Karger AG, Basel
\end{tabular} www.karger.com/cio

Afonso et al.: Ulnar Artery Cystic Adventitial Disease

\section{Materials \& Methods}

A 52-year-old right-hand-dominant female presented to our outpatient department for progressive paresthesia and dysesthesia along the fourth and fifth right fingers, with preserved sensitivity of the dorsum of the hand. History of repetitive hand and wrist trauma or previous surgeries were denied. Physical examination revealed a complete mobility of the wrist and fingers, however, some hand grip and adductor pollicis muscle weakness were noted when compared to the left hand. Froment's and Wartenberg's signs were positive, and Tinel's sign was present when tapping the ulnar nerve at Guyon's canal. MRI revealed a T2-weighted high-intensity lesion, measuring $29 \times 8 \times 4 \mathrm{~mm}$, and circumferentially surrounding the ulnar artery, without an identifiable pedicle connecting to the joint, and in close relation to the ulnar nerve over Guyon's canal (Fig. 1). An electromyographic study revealed a mild decrease in the left ulnar motor and sensory amplitudes at the level of the wrist.

The diagnosis of ulnar nerve compression at the wrist by a space-occupying lesion was made. Based on the MRI findings, an adventitial cyst and an aneurism of the ulnar artery were considered as differential diagnosis. Angiographic study or contrast-enhanced MRI could have demonstrated the definite diagnosis; however, given the motor impairment already present upon physical exam, surgical exploration was decided.

After interscalene brachial plexus block and general induction, without tourniquet, the patient right arm was prepped and draped. A 6-cm S-shaped volar incision crossing the wrist crease was used, and the flexor carpi ulnaris muscle retracted ulnarly after sharp dissection. The ulnar nerve and artery were isolated proximal to the pisiform and dissected distally, incising through the palmaris brevis and palmar carpal ligament, until identification of ulnar nerve bifurcation into its superficial and deep branches. The bulging of the ulnar artery was identified, beginning in zone 1 of Guyon's canal and extending until the ulnar nerve bifurcation (Fig. 2). Since the lesion appeared indissociable from the artery, resembling an aneurysm, we decided to prepare the ipsilateral forearm basilic vein for transposition, if needed, before further exploration. A single dose of 5,000 IU intravenous heparin was given, and the ulnar artery was clamped. A small longitudinal incision resulted in mucinous content extravasation, and through a meticulous dissection, the adventitia covering the lesion was removed without damage to the remaining vessel wall (Fig. 3). We were not able to identify
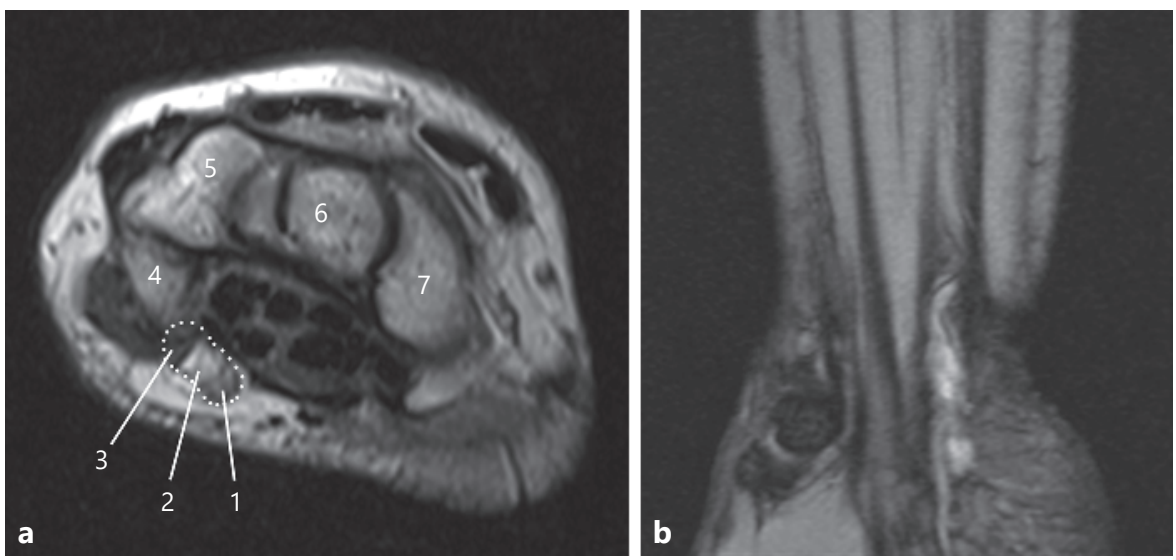

Fig. 1. a Sagittal T2-weighted magnetic resonance with fat suppression imaging showing high-intensity mass in Guyon's canal in close relation to the ulnar artery and compressing ulnar nerve. 1 - ulnar artery; 2 - adventitial cystic disease; 3 - ulnar nerve; 4 - pisiform; 5 - hamate; 6 - capitate; and 7 - scaphoid. b Coronal T2-weighted MRI showing the adventitial cyst of ulnar artery along its trajectory in Guyon's canal.

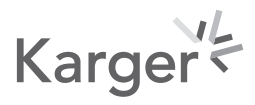




\section{Case Reports in Orthopedic Research}
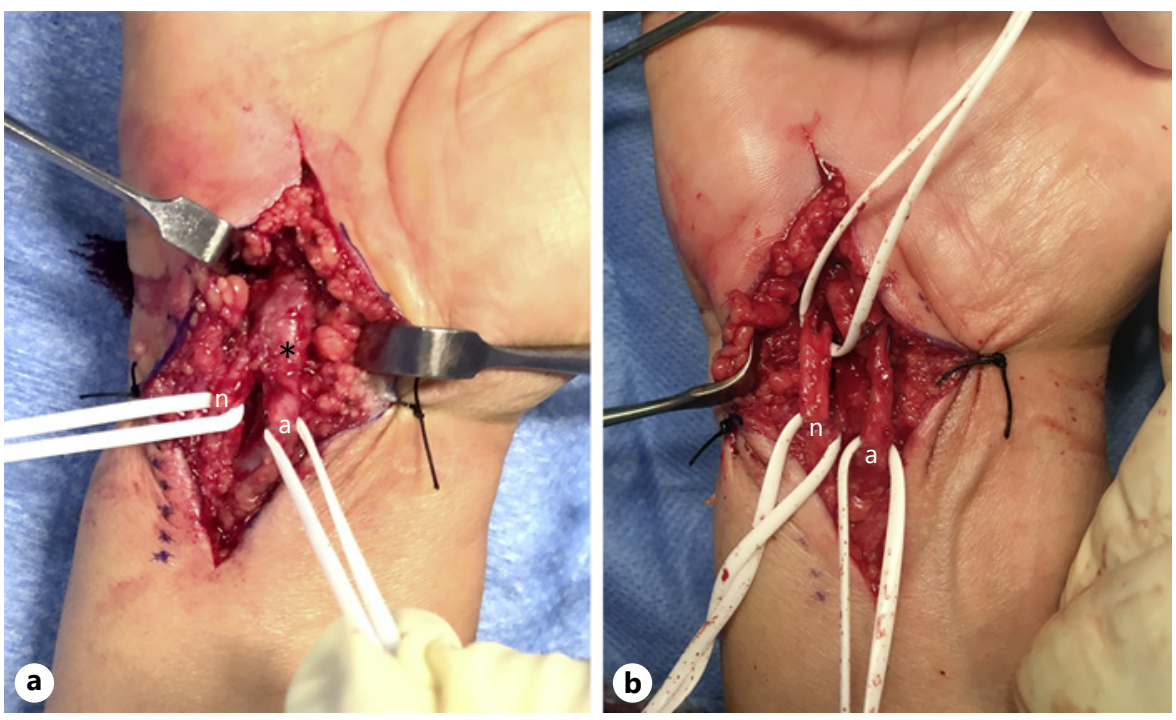

Fig. 2. a Intraoperative view of ulnar artery adventitial disease compressing the ulnar nerve. *, adventitial cystic disease; a, artery; $n$, nerve. b Intraoperative view after cyst excision and native artery wall preservation.

Fig. 3. Clinical result 6 months after surgery. Note the preserved fifth digit abduction strength and the absence of first dorsal interossei atrophy. Forment's sign was negative.

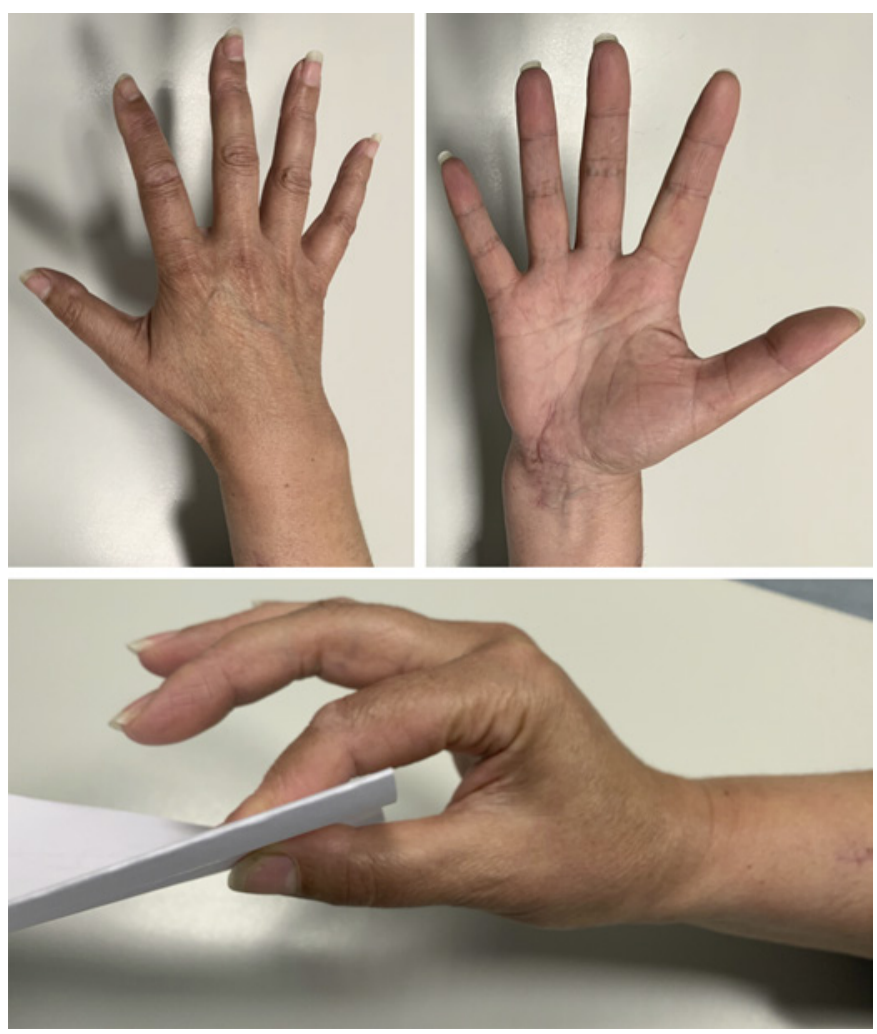

any pedicle connecting the cyst to the underlying wrist joints. After cyst excision, distal arterial patency was confirmed, the wound was irrigated, and the subcutaneous tissue and skin were closed in a standard fashion. Histopathological analysis confirmed the presence of a ganglion cyst. 
Four weeks after surgery, the patient referred complete paresthesia relief and progressive muscle strength improvement, until normalization at 6 months.

\section{Discussion}

Compression of the ulnar nerve can occur at multiple points along its course from the brachial plexus to its terminal branches at the level of the hand. Major sites of compression can occur at the elbow, specifically, Struthers arcade, cubital tunnel under the Osborne's ligament, and deep flexor pronator aponeurosis [3]. In rare occasions, the compression can present more distally, at the level of Guyon's canal, which is a unique semi-rigid anatomic structure, with a bone floor and fibrous roof, medially bounded by the pisiform and laterally by the hook of the hamate. The ulnar nerve and artery run close together into narrow Guyon's canal, being vulnerable to the effect of a space-occupying lesion in this area, such as ganglion cysts, tendinitis, fractures, arthritis, thromboangiitis, or aneurisms [4]. Gross and Gelberman [5] classified the ulnar-nerve compression syndromes in 3 clinically distinct types, in relation to the 3 zones previously described by Shea and McClain [6]. Zone 1 extends from the proximal edge of the palmar carpal ligament to the motor/sensory bifurcation. A combined motor and sensory deficit is expected if the compression occurs at this level. Zone 2 is ulnar and distal to the nerve bifurcation and includes the deep motor branch of the ulnar nerve. Compressive symptoms usually manifest as an isolated motor deficit. Zone 3 is more radial to the zone 2 and affects the superficial sensory branch of the ulnar nerve. Pure sensitive deficits are caused by its compression. Additionally, the dorsal cutaneous branch of the ulnar nerve emerges approximately $8.3 \mathrm{~cm}$ proximal to the pisiform, in order to innervate the dorso-ulnar side of the hand [7], which was spared in our patient. This fact, in addition to the combined motor and sensory deficits, pointed to a lesion at the level of the wrist, beginning at zone 1 of Guyon's canal to be more accurate. At this location, ganglion cysts are the most frequent cause of ulnar compression described in the literature [8]; however, they should not be confounded with an adventitial cyst of ulnar artery, an extremely rare condition, in which a mucin-containing cyst develops in the adventitial layer of the artery [1]. As a result, intraoperative macroscopic appearance resembles an aneurism (as represented in Fig. 2a).

Scientific knowledge about CAD is based on the cumulative data from around 500 cases reported in the literature, nevertheless, its underlying pathogenesis remains unclear. Several hypothesis have been postulated, with the "synovial or articular" theory gaining support in comparison to the "repetitive trauma" or "embryological" theories [1], especially after some studies reporting connections between adventitial cysts and adjacent joints. For unknown reasons, the majority of CAD occur in the popliteal artery, and in accordance, the scarce reviews available about CAD surgical treatment focused their attention on this vessel. Resection of the cyst and arterial segment with interposition vein graft, evacuation of the cyst with vein patch repair, or excision with preservation of native artery have been successfully used for popliteal artery CAD treatment. However, due to anatomical and caliber disparities, extrapolation of these results to a smaller caliber vessel would be inadequate. We were only able to find 2 case reports about CAD affecting the ulnar artery in Guyon's canal. The first one focused on the role of high-resolution ultrasound and MRI for CAD diagnosis, which is of paramount importance, anticipating potential difficulties during surgical approach [9]. More recently, Alba et al. [10] described a sole resection of the ulnar artery and its cystic disease since its entrance to Guyon's canal until the common digital artery of the third and fourth fingers. Due to hypoperfusion of the fifth finger, an interposition vein bypass from ulnar artery stump to the fourth and fifth common digital artery was needed. The location of the cyst within the vessel walls makes the risk of an iatrogenic lesion very high and occasionally

\section{Karger'}


it can even be indissociable from the artery. For that reason, it is essential that the surgeon to be familiarized with revascularization techniques, or alternatively, to be assisted by a vascular surgeon. Although less invasive approaches may be tried (e.g., ultrasonography-guided aspiration), we believe that due to the unique anatomy of Guyon's canal, surgical treatment may be considered the treatment of choice. This approach takes advantage of the cumulative effect related to lesion excision and tunnel decompression after cutting the palmaris brevis and palmar carpal ligament. In order to avoid the well-known disadvantages of autologous vein bypass (such as early thrombosis, intimal hyperplasia with stenosis/occlusion of the graft, or aneurysmal dilatation), we circumferentially removed the adventitial wall and its mucinous content, preserving the native artery. Nevertheless, it should not be forgotten that this technique weakens the artery wall with increased risk of aneurism formation, and because of that, these patients should be followed in a regular basis.

Ulnar nerve entrapment by a space-occupying lesion in Guyon's canal is a rare clinical condition, and a deep knowledge of ulnar nerve branches and its functions is needed in order to precisely determine the level of compression. Early lesion removal and tunnel decompression is crucial concerning the motor and sensory recovery.

\section{Statement of Ethics}

Written informed consent was obtained from the patient for publication of this case report and any accompanying images.

\section{Conflict of Interest Statement}

The authors have no conflicts of interest to declare.

\section{Funding Sources}

None to report.

\section{Author Contributions}

J.R.A.: involved in clinical evaluation of the patient, surgical planning, intervention, and follow-up; conception and design of the work; acquisition, analysis, and interpretation of the data; and drafting of the work. J.C.: conception and design of the work; acquisition, analysis, and interpretation of the data; and drafting of the work. M.Q.: conception and design of the work; acquisition, analysis, and interpretation of the data; and drafting of the work. J.Va.: involved in surgical intervention, analysis and interpretation of the data, and reviewed the manuscript. J.Vi.: analysis and interpretation of the data and reviewed the manuscript. D.B.L.: analysis and interpretation of the data and reviewed the manuscript. C.V.: involved in surgical intervention, analysis and interpretation of the data, and reviewed the manuscript.

\section{Karger'k}




\section{References}

1 Desy NM, Spinner RJ. The etiology and management of cystic adventitial disease. J Vasc Surg. 2014;60(1): 235-45, 45 e1-11.

2 Atkins HJ, Key JA. A case of myxomatous tumour arising in the adventitia of the left external iliac artery; case report. Br J Surg. 1947;34(136):426.

3 Earp BE, Floyd WE, Louie D, Koris M, Protomastro P. Ulnar nerve entrapment at the wrist. J Am Acad Orthop Surg. 2014;22(11):699-706.

4 Chen SH, Tsai TM. Ulnar tunnel syndrome. J Hand Surg Am. 2014;39(3):571-9.

5 Gross MS, Gelberman RH. The anatomy of the distal ulnar tunnel. Clin Orthop Relat Res. 1985;(196):238-47.

6 Shea JD, McClain EJ. Ulnar-nerve compression syndromes at and below the wrist. J Bone Joint Surg Am. 1969; 51(6):1095-103.

7 Goto A, Kunihiro 0, Murase T, Moritomo H. The dorsal cutaneous branch of the ulnar nerve: an anatomical study. Hand Surg. 2010;15(3):165-8.

8 Kuschner SH, Gelberman RH, Jennings C. Ulnar nerve compression at the wrist. J Hand Surg Am. 1988;13(4): 577-80.

9 Pivec C, Sillat T, Moritz T, Riegler G, Nanobachvili J, Bodner G. A rare case of Guyon's canal syndrome caused by cystic adventitia degeneration: high-resolution ultrasound findings. Ultraschall Med. 2017;38(5):556-7.

10 Alba JAP. Adventitial cyst in ulner artery and palmar superficial arch of the hand: a case report. Acta Sci Orthop. 2020;3(4):33-7. 